\title{
Tissue-dependence and sensitivity of the systemic RNA interference response in the desert locust, Schistocerca gregaria
}

\author{
Niels Wynant*, Heleen Verlinden, Bert Breugelmans, Gert Simonet, Jozef Vanden Broeck \\ Molecular Developmental Physiology and Signal Transduction, KU Leuven, Naamsestraat 59, P.O. Box 02465, B-3000 Leuven, Belgium
}

\section{A R T I C L E I N F O}

\section{Article history:}

Received 29 June 2012

Received in revised form

10 September 2012

Accepted 13 September 2012

\begin{abstract}
A B S T R A C T
We report on a comprehensive study of the systemic RNAi-response in the desert locust, Schistocerca gregaria. Upon intra-abdominal injection of dsRNA for two housekeeping genes (alpha-tubulin $1 a$ and gapdh) in the range of pg amounts of dsRNA per mg tissue, a potent reduction of their corresponding mRNA was obtained. Moreover, the observed transcript knockdown significantly increased in at least a 10 days period and eventually resulted in high mortality upon silencing of the alpha-tubulin 1a gene. A more moderate RNAi-response was however observed in the reproductive systems. Analysis of the tissuedependent transcript level profile of several putative RNAi-genes indicated reduced levels of two genes, namely sg-dicer-2 and sg-argonaute-2, in the reproductive systems. By silencing these components, we confirmed their importance in the RNAi-process and suggest that their expression levels are determinant for tissue-dependent differences in the potency of RNAi in the desert locust.
\end{abstract}

(c) 2012 Elsevier Ltd. All rights reserved.

\section{Introduction}

Several studies have shown that double stranded (ds)RNA can initiate the activation of an innate immune response, resulting in the sequence-specific inhibition of cellular gene expression at the post-transcriptional level (Hammond et al., 2001). This process, better known as RNA interference (RNAi), has become a widely used reverse genetic tool to knockdown and analyse the function of genes. Besides functioning as a research tool, RNAi may also contribute to novel strategies for selectively controlling agricultural pests, including a number of insect species (Mao et al., 2007; Baum et al., 2007; Whyard et al., 2009). In contrast to conventional insecticides, RNAi is a nucleic acid sequence dependent process. Consequently, RNAi is a highly species-specific technology, the use of which should result in fewer non-target effects and reduced harm to agricultural ecosystems. However, in order to induce an RNAi-response, dsRNA must enter the cell. Fortunately, some organisms are able to import extracellular dsRNA via dsRNAspecific transporters. One well-known uptake mechanism is transmembrane channel-mediated dsRNA-transport exemplified by the Caenorhabditis elegans SID-1 protein (Winston et al., 2002). A second uptake pathway was discovered in Drosophila melanogaster Schneider 2 (S2) cells, which employ receptor-mediated

\footnotetext{
* Corresponding author. Tel.: +32 16324260; fax: +32 16323902.

E-mail address: Niels.Wynant@bio.kuleuven.be (N. Wynant).
}

endocytosis (Ulvila et al., 2006; Saleh et al., 2006). In addition, in several plants and nematodes, secondary dsRNA molecules are produced by RNA dependent RNA Polymerases (RdRP), whereby even with the presence of very low dsRNA amounts, a robust and persistent RNAi-response will be achieved (Sijen et al., 2001; Schiebel et al., 1998). However, until now no canonical RdRP orthologous sequences have been found in the genome of insects (Belles, 2010).

In many insect species, representing the orders of Lepidoptera, Coleoptera, Diptera, Hemiptera, Hymenoptera, Dictyoptera, Isoptera and Orthoptera, systemic RNAi-responses have been reported (Belles, 2010). However, the degree of sensitivity toward RNAi varies strongly among insect species (Miller et al., 2008; Belles, 2010; Terenius et al., 2011). The best-known example of a species with a reduced sensitivity towards systemic RNAi is the fruit fly D. melanogaster (Miller et al., 2008). In its larval stage, only the hemocytes seem to be capable of responding in a systemic manner. Furthermore, in many Lepidoptera, such as Bombyx mori and Spodoptera littoralis, most tissues also seem to be rather refractory to a systemic response (Terenius et al., 2011). On the other hand, recent reports suggest that some Orthoptera, Dictyoptera and Coleoptera respond very well to systemically applied dsRNA (Badisco et al., 2011b; Dong and Friedrich, 2005; Belles, 2010; Miller et al., 2008). Here, we report on the first detailed description of the systemic RNAi-response in a member of the Orthoptera, namely Schistocera gregaria, a voracious pest insect and an important research organism. Although previous studies have proven the 
presence of a robust systemic RNAi-response in S. gregaria (Badisco et al., 2011b; Marchal et al., 2011a, b; Ott et al., 2012; Van Wielendaele et al., 2012; Marchal et al., 2012), its sensitivity, persistence and tissue dependency have not been investigated in detail so far. In this study, we demonstrate that injection of nanomole amounts of dsRNA can silence up to $90 \%$ of the gene transcript level, with a knockdown effectiveness that increases over a ten-day period. Furthermore, a clearly reduced susceptibility towards RNAi was observed in the reproductive system, which may be caused by lower expression levels of components of the core RNAi-machinery.

\section{Materials and methods}

\subsection{Rearing of Schistocerca gregaria}

Gregarious S. gregaria were reared under crowded conditions with controlled temperature $\left(32 \pm 1{ }^{\circ} \mathrm{C}\right)$, light ( $13 \mathrm{~h}$ photoperiod) and relative humidity (40-60\%). They were fed daily with fresh cabbage and dried oat flakes. Directly after their final moult, locusts were synchronized to obtain adult animals of equal age.

\subsection{Sequence information}

Transcript sequence information of $S$. gregaria glyceraldehyde phosphate dehydrogenase (gapdh) (GenBank: HQ851387), alphatubulin 1a (GenBank: HQ851397), dicer-2 (dcr2) (GenBank: JX516787), argonaute-2 (ago2) (GenBank: JX516788), enhanced RNA interference family member (eri1) (GenBank: JX516790) was retrieved from the annotated $S$. gregraria EST-database (Badisco et al., 2011a). There was no sequence available for the sg-sid1 transcript. However, based on the orthologous sequence found in S. americana by Dong and Friedrich (2005), a fragment of $435 \mathrm{bp}$ was amplified (GenBank: JX516789). The DNA fragments were cloned into the $\mathrm{pCR}^{\circledR} 4$-TOPO ${ }^{\circledR}$ vector by means of the TOPO TA Cloning ${ }^{\circledR}$ Kit for Sequencing (Invitrogen). The sequences of the inserted DNA fragments were determined using the ABI PRISM BigDye Terminator Ready Reaction Cycle Sequencing Kit (Applied Biosystems). The obtained sequences were subsequently compared with BLAST to NCBI nucleotide databases of other insects and in silico translated into the corresponding amino acid sequences. The latter were then aligned against orthologous sequences of other insects (ClustalW), and the predicted protein domains were identified (NCBI, CD-search). In addition, since Dicer-1 (Dcr1) and Argonaute-1 (Ago1) are related to Dcr2 and Ago2 proteins, respectively, we have constructed an unrooted phylogenetic tree (ExPASy, ETH Zurich) to determine the correct identity of the sequences of $S$. gregaria (Supplementary data in Fig. S3).

\subsection{Synthesis of dsRNA}

Double stranded RNAs for alpha-tubulin 1a (545 bp), gapdh (447 bp), gfp (589 bp), dcr2 (330 bp) and ago2 (461 bp) were synthesised using the MEGAscript RNAi kit (Ambion). For production of alpha-tubulin 1a, gapdh, dcr2 and ago2 dsRNA, a DNA template flanked by two T7 promoter sequences was generated. A PCR reaction was performed using adult desert locust cDNA and gene specific primers containing a $\mathrm{T} 7$ promoter sequence at the $5^{\prime}$ end. In the supplementary data (Fig. S1), the different PCR primers are displayed. REDTaq mix (Sigma-Aldrich co.) was used as a source of DNA Taq polymerase, dNTPs and PCR buffer. Next, the amplification products were analysed by $1 \%$ agarose gel electrophoresis and then visualised with UV-light. Finally, bands of the desired size were cut out and purified using GenElute ${ }^{\mathrm{TM}}$ Gel extraction Kit (SigmaAldrich Co.). A TOPO 4.1 sequencing vector (Invitrogen) containing a gfp transcript sequence was used as template for gfp dsRNA production. Since only one T7 promoter site is present in this vector, the fragment was cloned both in the sense and antisense direction. RNA was then synthesised by the T7 Enzyme Mix of the MEGAscript RNAi kit (Ambion). Both gfp RNA strands were first synthesised independently before being mixed to anneal, while transcripts made from a single template with opposing T7 promotors were hybridized during the transcription reaction. After the production of dsRNA, the remaining DNA and ssRNA was removed by nuclease treatment, and proteins and mono/oligonucleotides were removed by solid phase adsorption purification, according to the manufacturers' specifications (Ambion). The dsRNAconcentration was determined with a Nanodrop spectrophotometer (Thermo Fisher Scientific, Inc.), and the integrity of the dsRNA was assessed with gel electrophoresis using a $1 \%$ agarose gel. The dsRNA was stored at $-20^{\circ} \mathrm{C}$ until further usage.

\subsection{Injection and tissue collection of adult locusts}

S. gregaria Ringer solution ( $1 \mathrm{~L}: 8.766 \mathrm{~g} \mathrm{NaCl} ; 0.188 \mathrm{~g} \mathrm{CaCl}_{2}$; 0.746 g KCl; $0.407 \mathrm{~g} \mathrm{MgCl}_{2} ; 0.336$ g NaHCO${ }_{3} ; 30.807$ g sucrose; $1.892 \mathrm{~g}$ trehalose; $\mathrm{pH}$ 7.2) was used to dilute the dsRNA to the desired concentration. Locusts were then each injected with $10 \mu \mathrm{l}$ dsRNA-solution. The locust tissues of interest were micro-dissected in S. gregaria Ringer solution under a binocular microscope and immediately transferred to liquid nitrogen to prevent RNA degradation. All samples were stored at $-80^{\circ} \mathrm{C}$ until further processing.

\subsection{RNA extraction and cDNA synthesis}

The Lipid tissue extraction kit (Qiagen) was utilized to extract RNA from larger tissues, such as gut, gonadal, muscle and fat tissue, as well as all pooled samples, while the RNAaqeous ${ }^{\circledR}$-micro kit (Ambion) was employed to extract RNA from the relatively smaller brain and malpighian tubules. This was followed by an additional DNase treatment to remove genomic DNA contamination. Quality and concentration of the extracted RNA were assessed using a Nanodrop spectrophotometer. Next, equal amounts of RNA were used as template to produce cDNA. The cDNA synthesis was performed using the Superscript III reverse transcriptase kit (Invitrogen), random hexamer primers (Invitrogen) and dNTPs (Roche diagnostics $\mathrm{GmbH}$ ) as described in the corresponding protocol. Finally, the cDNA-solution was 10-times diluted with MilliQ water. A calibrator cDNA sample was also made based on a pooled tissue sample containing all investigated tissues.

\subsection{Quantitative real time-PCR}

Primer express software (Applied Biosystems) was used to design quantitative real time (RT-)PCR primers. The corresponding primer sequences are displayed as supplementary data in Fig. S2. The primers were validated with a standard curve based on a serial dilution of cDNA to determine the primer annealing efficiency, and a dissociation protocol was performed to detect the presence of primer dimers and production of a single PCR product. For all transcripts, only a single melting peak was found. In addition, the qRT-PCR products were run on a $1 \%$ agarose gel containing GelRedTM (Biotium) that resulted in a single band. Each reaction was performed in duplicate and contained $10 \mu \mathrm{l} \mathrm{SYBR}$ green solution (Invitrogen), $0.75 \mu \mathrm{l}$ of $10 \mu \mathrm{M}$ forward primer (Sigma-Aldrich), $0.75 \mu \mathrm{l}$ of $10 \mu \mathrm{M}$ reverse primer (Sigma-Aldrich), $3.5 \mu \mathrm{l}$ milliQ water and $5 \mu \mathrm{l}$ cDNA. Seven household genes were chosen, based on the study of Van Hiel and co-workers (Van Hiel et al., 2009). The geNorm program (Pattyn et al., 2003) was used to determine the most stable reference genes in the designed experiments. The four genes that were most stably expressed were, respectively, ubiquitin 
conjugating enzyme 10 (ubi), elongation factor $1 a$ (ef1a), gapdh and alpha-tubulin 1a. In order to correct for sample-to-sample variations, the relative expression levels were normalized against the two most stably expressed reference genes, $u b i$ and ef $1 a$. The data were further normalized against a calibrator cDNA sample to account for variations in the PCR-efficiency in different PCR runs. In every experiment, no-template and no-RT controls were included to check for possible contaminations. The PCR reaction was performed and analysed in a 96 well plate and by the StepOne System (ABI Prism, Applied Biosystems). Since the efficiency of the different primers was the same, the relative transcript quantity was calculated according to the delta-delta $\mathrm{Ct}$ method.

\subsection{Statistical analysis}

All data were analysed by non-parametric statistics in GraphPad prism 5 (GraphPad). In addition, the change of transcript knockdown efficiency over time (Section 3.2) was assessed by linear regression analysis.

\section{Results}

\subsection{Tissue-dependence of the systemic RNAi-response}

Five $\mu \mathrm{g}$ of $s g$-gapdh dsRNA was injected into the haemocoel of four-day old adult locusts. Three days later, the tissue-dependent knockdown was determined by comparing the relative gapdh transcript level between the test and control group, i.e. locusts injected with gfp dsRNA. The data (Fig. 1) indicate that a very robust knockdown was achieved for most tissues investigated, with the exception of the male and female reproductive systems. While a significant knockdown was still observed in the reproductive system, it was tempered in comparison to the other tissues investigated.

\section{2. dsRNA-dose and time dependence of the RNAi-response}

The dsRNA-dose dependence was studied by intra-abdominal injection using various amounts of gapdh dsRNA. After three days, the knockdown for two distinct tissues was examined: the female reproductive system, which is known to be more resistant towards RNAi, and the brain, which is one of the more responsive tissues (Section 3.1). The data demonstrate that the RNAi-response was extremely sensitive (Fig. 2a). Injection of only $1 \mathrm{ng}$ of gapdh dsRNA

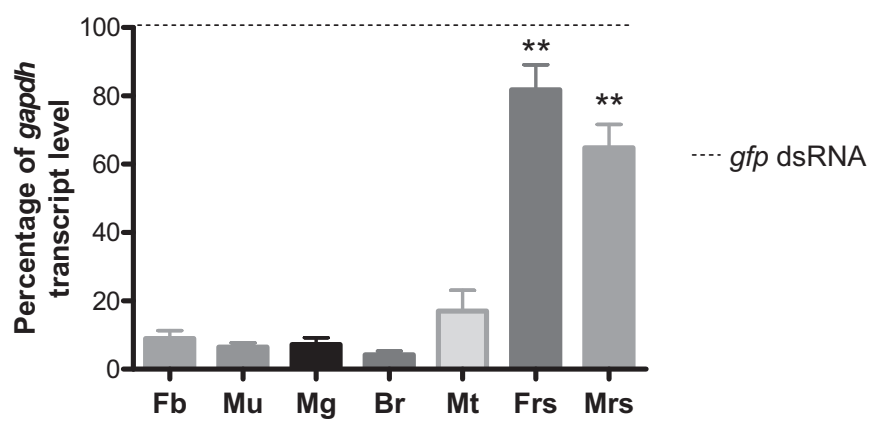

Fig. 1. Tissue-dependence of systemic RNAi, three days after injection of $5 \mu \mathrm{g}$ of dsRNA. Seven different tissues were examined: $\mathrm{Fb}$ (fatbody), Mu (muscles), Mg (midgut), Br (brain), Mt (malpighian tubules), Frs (Female reproductive system) and Mrs (Male reproductive system). Significantly higher transcript levels were observed in the Mrs and Frs. The knockdown is represented as the mean percentage of the relative gapdh transcript levels in the test group in comparison to the mean relative transcript levels in the control groups that were injected with gfp dsRNA (mean \pm SEM, $n \geq 5$, $* *: p<0.01)$.
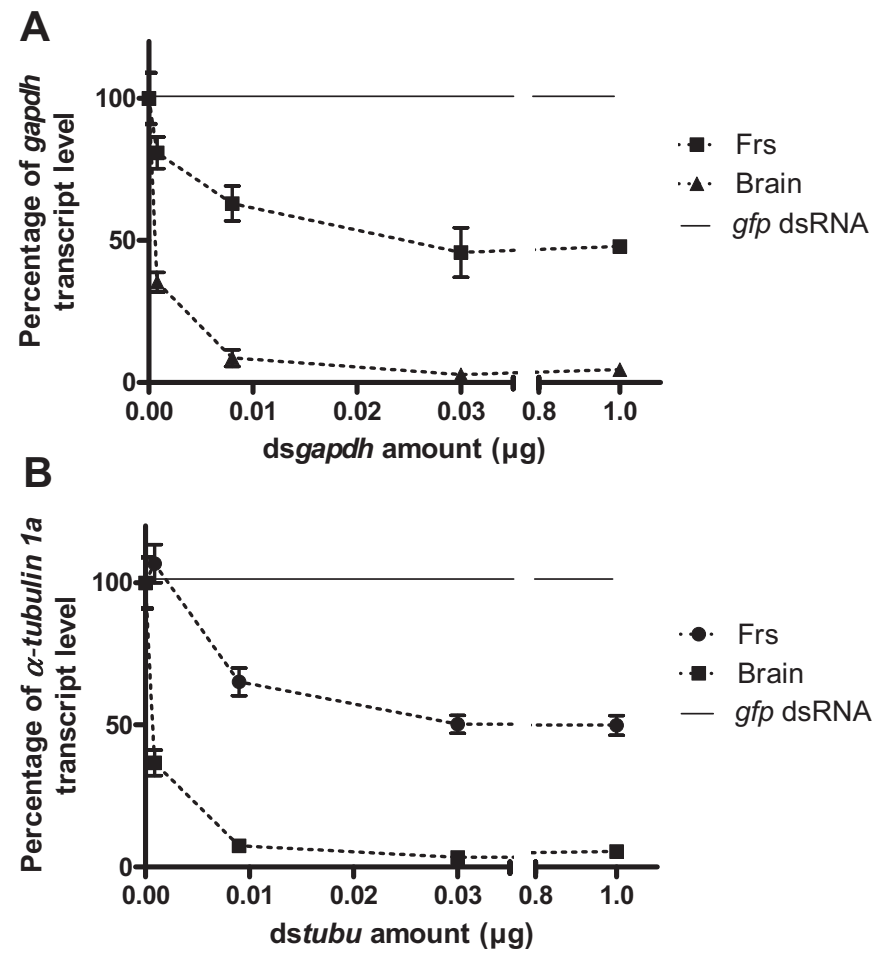

Fig. 2. RNAi-response three days after injection of different amounts of dsRNA for (a) gapdh and (b) $\alpha$-tubuline $1 a$ in the Female reproductive system (Frs) and brain. The knockdown is displayed as the mean percentage of the transcript levels present in the test groups in comparison to the mean relative transcript levels in the control groups that were injected with $g f p$ dsRNA (mean $\pm \operatorname{SEM}, n \geq 4$ ).

was sufficient to induce a significant knockdown. Moreover, the maximum concentration dependent silencing was observed upon administration of as little as 30 ng dsRNA, an amount that corresponds to an average of $15 \mathrm{pg}$ per mg tissue. Remarkably, despite the reduced knockdown robustness in the female reproductive system, both tissues displayed a similar dsRNA-dose-response relationship. To ascertain that these observations were not specific for gapdh gene silencing, a similar experiment was conducted using alpha-tubulin 1a dsRNA. Silencing of alpha-tubulin 1a resulted in a similar dose-dependent response (Fig. 2b), suggesting that the reported sensitivity is not specific to the gapdh gene or dsRNA sequence.

Similarly, by measuring the knockdown after three different durations; 1 -day, 3-days and 10-days post injection (p.i.), the timedependency of the RNAi-response was assessed. Five $\mu \mathrm{g}$ gapdh dsRNA was applied to saturate the RNAi-machinery, and four different tissues were examined, brain and midgut representing the more responsive tissues and male and female reproductive systems as less robust responders (Section 3.1). Our data show that the silencing response increased significantly with time, for all four tissues (Fig. 3a). A severe knockdown was seen 1-day p.i. in the brain and midgut, while this was minimal at this time point in the reproductive tissues. Similar time dependence was observed for alpha-tubulin 1a (Fig. 3b). However, in contrast to silencing the gapdh gene, injection of alpha-tubulin 1a dsRNA resulted in locust mortality, with the bulk of the locust population being dead 14days p.i.. Since initial lethality occurred 7-days after injection of the dsRNA, the latest assessed time point for alpha-tubulin 1a was 6 -days, instead of 10-days p.i..

The transcript levels of alpha-tubulin $1 a$ and gapdh in midgut, brain, female reproductive system and male reproductive system 

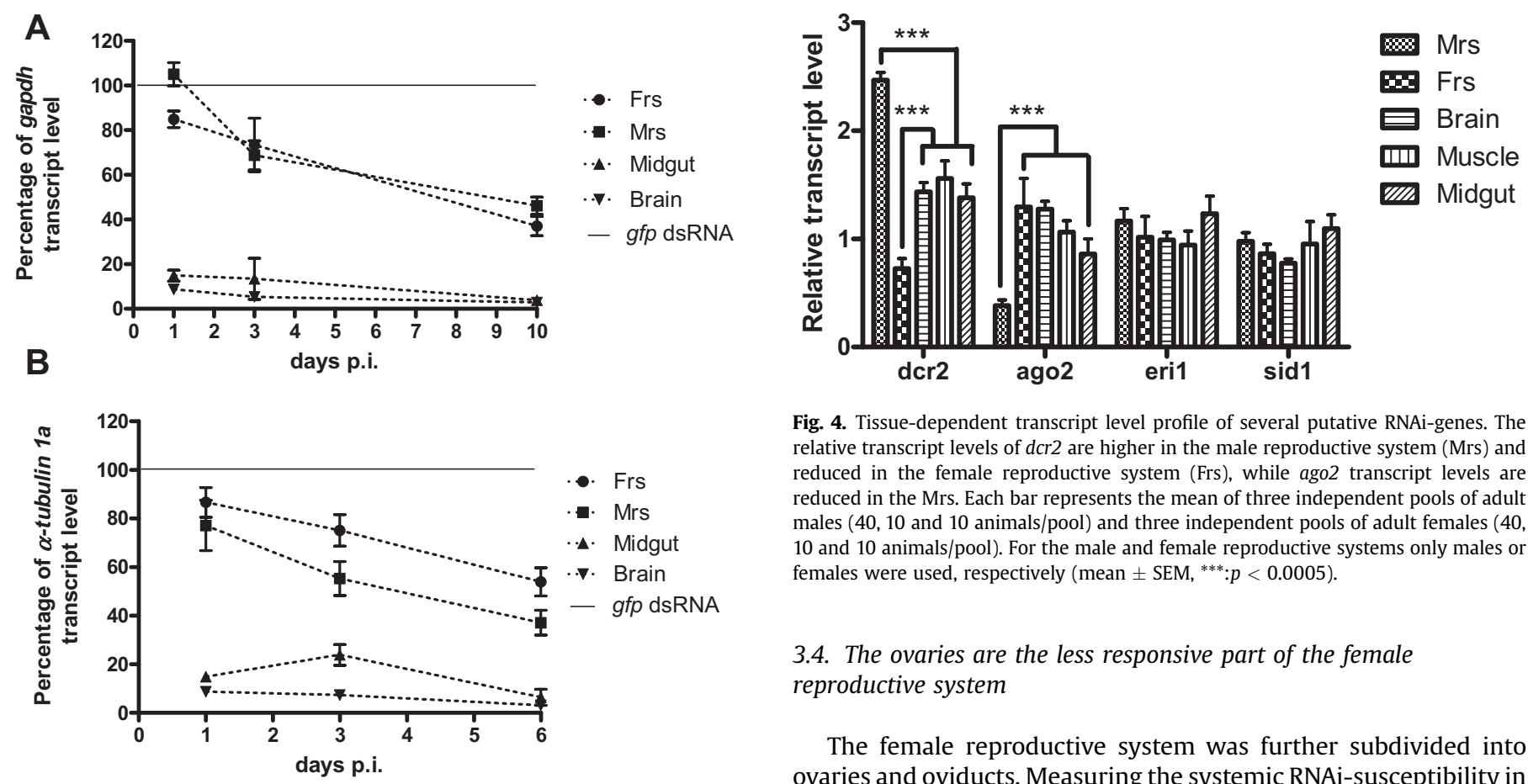

Fig. 4. Tissue-dependent transcript level profile of several putative RNAi-genes. The relative transcript levels of $d c r 2$ are higher in the male reproductive system (Mrs) and reduced in the female reproductive system (Frs), while ago2 transcript levels are reduced in the Mrs. Each bar represents the mean of three independent pools of adult males (40, 10 and 10 animals/pool) and three independent pools of adult females (40, 10 and 10 animals/pool). For the male and female reproductive systems only males or females were used, respectively (mean \pm SEM, ${ }^{* * *}: p<0.0005$ ).

\subsection{The ovaries are the less responsive part of the female reproductive system}

The female reproductive system was further subdivided into ovaries and oviducts. Measuring the systemic RNAi-susceptibility in these tissues revealed a very strong knockdown in the oviducts, while a significantly lower knockdown was obtained in the ovaries (Fig. 5a). Next, we tested if the reduced dcr2 expression level in the female reproductive system is also limited to the ovaries. The expression levels of $d c r 2$ and ago 2 were measured, and as displayed in comparison to the mean relative transcript levels in the control groups that were injected with gfp dsRNA, i.e. at each time point (mean \pm SEM, $n \geq 6$ ). The gapdh transcript levels decreases in all four tissues in course of time (Frs: $p<0.0001$, Mrs: $p<0.0001$, midgut: $p<0.005$ and brain: $p<0.0005)$. For $\alpha$-tubulin $1 a$ the transcript amount decreases in the Frs $(p<0.001)$, the $\operatorname{Mrs}(p<0.005)$ and the brain $(p<0.005)$.

can be found as supplementary data in Fig. S4. This demonstrates that the observed differences in RNAi-potency are not due to significantly higher expression levels of these genes.

\subsection{The reproductive system differentially expresses ago2 and dcr2 transcript levels}

In order to investigate the underlying causality of the reduced RNAi-susceptiblity in the reproductive system, we assessed the tissue-dependent transcript profile of several putative components of the RNAi-process. The systemic RNAi-process can be subdivided in two steps: first, the transport and spreading of the dsRNA into the tissues, and secondly, the cell-autonomous RNAi-response exerted by the core RNAi-machinery. We tested if different tissues show differences in expression levels for genes involved in these processes. As previously mentioned, SID-1 is a putative dsRNAtransporter (Winston et al., 2002). Furthermore, two genes well known to play a crucial role in the core RNAi-machinery of $D$. melanogaster and Tribolium castaneum are dcr2 and ago2 (Tomoyasu et al., 2008; Tomari and Zamore, 2005). As pointed out by several reports (Kennedy et al., 2004), dsRNA-degrading enzymes, such as ERI-1, may also negatively regulate the silencing process. Therefore, we measured the tissue-distribution of $d c r 2$, ago2, sid 1 and eri1 transcript levels in four-day old S. gregaria. Our results (Fig. 4) show that the transcript levels of $d c r 2$ were relatively lower in female and higher in male reproductive organs, while significantly lower ago2 expression levels were observed in the male reproductive system. On the other hand, the transcript levels of sid1 and eri1 remained indistinguishable between the different tissues investigated.

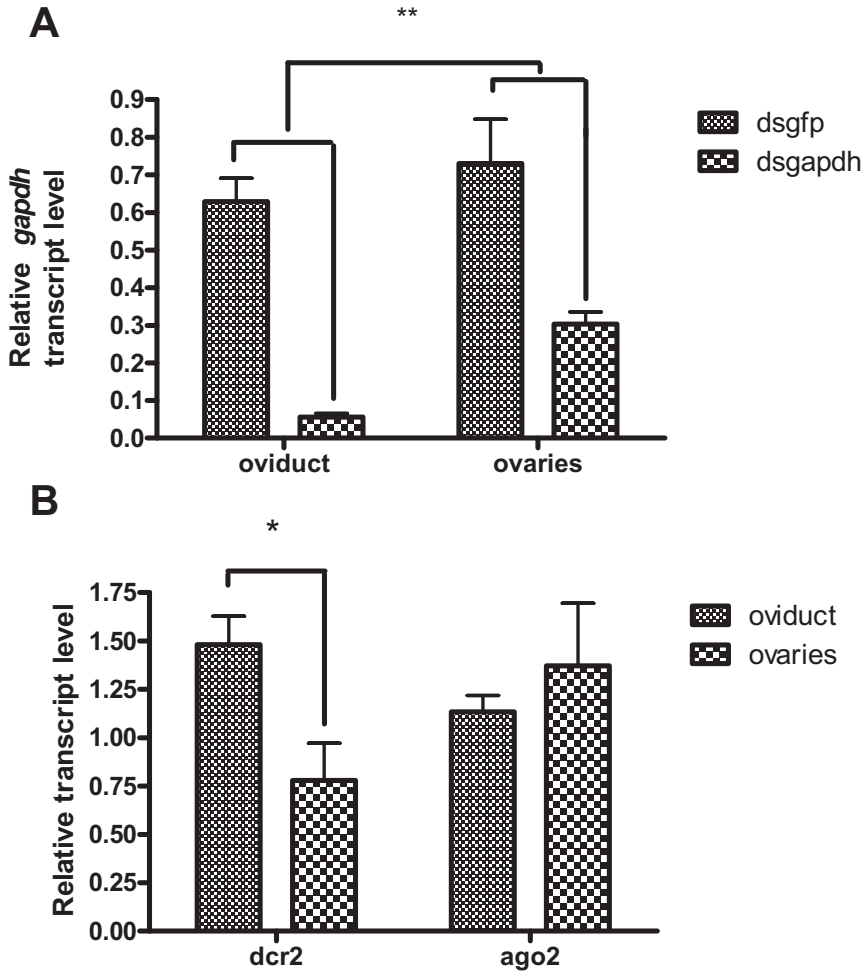

Fig. 5. Reduced RNAi-susceptibility and lower transcript levels of $d c r 2$ in the ovaries in comparison to the oviducts. (a) Knockdown of gapdh in oviducts and ovaries (mean \pm SEM, $n \geq 6,{ }^{* *}: p<0.01$ ). (b) Relative transcript levels of $d c r 2$ and ago2 in the oviducts and ovaries (mean \pm SEM, $n \geq 6$, ${ }^{*}: p<0.05$ ). 
in Fig. 5b the ovaries contain relatively less dcr2 transcript levels, while no significant differences could be reported for ago2.

\subsection{Lower dcr2 or ago2 transcript levels result in reduced RNAi- susceptibility}

In order to investigate whether the expression levels of either dcr2 or ago2 can influence the RNAi-susceptibility, we silenced these components by injecting $100 \mathrm{ng}$ of gene specific dsRNA. As a control, 100 ng of $g f p$ dsRNA was injected. Six days later, a second injection was performed with $100 \mathrm{ng}$ of alpha-tubulin $1 a$ dsRNA. To be able to determine the knockdown effectiveness, a second control group was taken into account that was treated with a double $g f p$ dsRNA injection. The data in Fig. 6a show a successful knockdown of ago 2 and dcr2 in midgut tissue and, as demonstrated in Fig. 6b. this resulted in a reduced knockdown of the alpha-tubulin $1 a$ gene
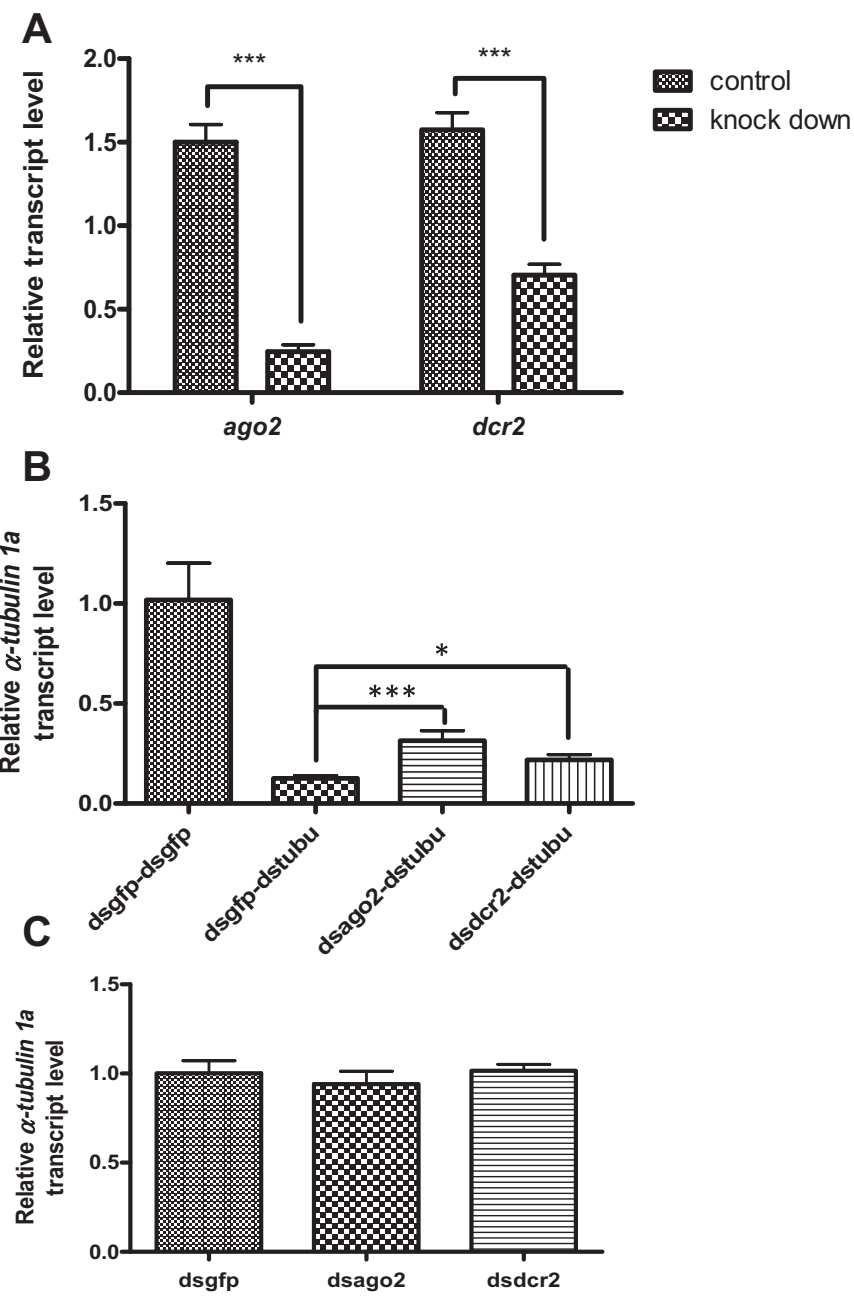

Fig. 6. Both ago 2 and $d c r 2$ are involved in the RNAi-pathway. (a) An effective knockdown of ago2 and dcr 2 transcript levels was obtained (mean $\pm \operatorname{SEM}, n \geq 6$; ${ }^{* * *}$; $p<0.001$ ). (b) The locusts were injected with either $100 \mathrm{ng}$ of dsgfp, dsago2 or dsdcr2 and six days later followed by a second dsRNA injection of $100 \mathrm{ng}$ of alpha-tubulin $1 \mathrm{a}$ (dstubu), indicated as respectively dsgfp-dstubu, dsago2-dstubu and dsdcr2-dstubu. In addition, a second control group that was twice injected with dsgfp, indicated as dsgfp-dsgfp, was also assessed. Fourteen hours after the second injection midgut tissue was microdissected and the relative transcript levels were then measured with real-time PCR. Statistical analysis was performed between the dsago2-dstubu or dsdcr2-dstubu group and the group dsgfp-dstubu (mean \pm SEM, $n \geq 8,{ }^{* * *}: p<0.001$ and $* ; p<05$ ). (c) Silencing of ago2 or $d c r 2$ transcript levels had no direct effect on the alpha-tubulin $1 a$ transcript level in the midgut. The expression level was determined 6 days after injection of dsgfp, dsago2 or dsdcr2 (mean $\pm \operatorname{SEM}, n \geq 5$ ). expression. It is, however, possible that silencing dcr2 or ago 2 directly influences the alpha-tubulin $1 a$ gene expression level, rather than the RNAi-susceptibility. Therefore, the alpha-tubulin 1a expression level was also determined 6 days after injection of $d c r 2$ or ago2 dsRNA, without injection of alpha-tubulin 1a dsRNA. As demonstrated in Fig. 6c, no differential alpha-tubulin 1a transcript levels were reported.

\section{Discussion}

In this paper, we report on a detailed examination of the systemic RNAi-response in the desert locust, S. gregaria. Our results demonstrate that, in contrast to the relatively high resistance against systemic RNAi in many dipteran and lepidopteran species (Terenius et al., 2011; Miller et al., 2008; Belles, 2010), application of as little as 15 pg of dsRNA per mg tissue is sufficient to almost completely silence specific gene expression in adult desert locusts (Fig. 2). This finding is in accordance with the recently published highly sensitive RNAi-response in the migratory locust, Locusta migratoria (Luo et al., 2012). In addition, we show that the reduction in transcript levels persists for a long time, with an increase in knockdown efficacy during (at least) a ten-day period (Fig. 3), thereby rendering the desert locust an extremely RNAi-sensitive and -persistent insect species. Targeting the alpha-tubulin $1 a$ mRNA gene product resulted in mortality in the locusts' population (Section 3.2), confirming knockdown at the protein level and suggesting that RNAi may contribute to promising strategies for selectively controlling locust pests. Its highly effective systemic RNAi-response, in combination with the available S. gregaria ESTsequence information (Badisco et al., 2011a), makes the desert locust an attractive organism for 'loss of function' analyses.

On the other hand, a more moderate RNAi-response is observed in the male and female reproductive systems (Fig. 1). This effect is most pronounced shortly after injection of dsRNA, but eventually also a clear knockdown effect will be reached in these organs (Fig. 3). By investigating the tissue-dependent transcript profile of several putative RNAi-genes, we assessed the possible influence of (a) the core RNAi-machinery, (b) dsRNAtransport from the hemolymph into the tissue, and finally, (c) dsRNA-degradation in the tissues. This analysis revealed reduced dcr2 and ago2 transcript levels in the female and male reproductive systems, respectively (Fig. 4). Since their function in locusts was still unclear, we silenced dcr2 and ago 2 and confirmed their role in the RNAi-process in S. gregaria (Fig. 6). This is in agreement with the role of these genes in D. melanogaster and T. castaneum (Tomoyasu et al., 2008; Lee et al., 2004; Czech et al., 2009), and is the first report on their function in a member of the Orthoptera. These components clearly play a crucial role in the core RNAipathway of D. melanogaster (Kim et al., 2006). However, silencing dcr2 or ago2 in the desert locust did not completely abolish the RNAi-response (Fig. 6). This should not necessarily imply that their involvement in RNAi is less important in $S$. gregaria. First, RNAi only generates a silencing effect at the posttranscriptional level. Second, previous studies have demonstrated that the protein turnover rate can be a determinate factor for the potency of the knockdown at the (functional) protein level (Ott et al., 2012). A slow protein turnover rate may thus account for a rather moderate functional effect of the RNAi-response.

Although both male and female reproductive systems display less potent knockdown effects, their reported transcript profiles for the RNAi-genes ago2 and dcr2 are dissimilar. Whereas dcr2 transcript levels are lower in the female reproductive system, the male reproductive system contains less ago 2 and more $d c r 2$ transcripts (Fig. 4). This finding illustrates the complexity of RNAi and indicates that the process may be regulated differently in different tissues. 
The reasons for this are currently not clear. One possible explanation could reside in the fact that, as reported in $D$. melanogaster, some cross-talk and overlap may exist between the short interference (si)RNA- and micro (mi)RNA-pathways (Zhou et al., 2008; Tomari et al., 2007). Whereas the siRNA-pathway is primarily an anti-viral immune response that recognizes dsRNA-molecules, the miRNA-pathway regulates the expression of endogenously produced mRNA-targets (Carthew and Sontheimer, 2009). This could mean that regulation of the expression level of components of the (si)RNAi-pathway might also affect the miRNA-pathway, or vice versa, and that compensatory control mechanisms might be required. An analysis of the components of the miRNA-pathway would therefore be of interest to verify this hypothesis. Unfortunately, sequence information of these components was either not available in the S. gregaria EST-database, or the fragments were too short to design suitable qRT-PCR primers. Nevertheless, it remains remarkable that specifically in the reproductive system a more moderate RNAi-potency is observed and that this coincides with differential transcript levels of $d c r 2$ or ago2. Since silencing either dcr 2 or ago 2 is sufficient to reduce the knockdown efficiency (Fig. 6), reduced transcript levels of one of these components may account for a lower RNAi-potency. It is therefore probable that each of these enzymes represents a rate-limiting step for the RNAiprocess. However, it remains unclear to what extent an increase of $d c r 2$ transcripts (as observed in the male reproductive system) could (partially) undo the effects caused by lower ago 2 transcript levels.

Interestingly, low transcript levels of the core RNAicomponents, Bm-R2D2, Ms-dcr2 and Ms-ago2, have previously been considered as an explanation for the apparent inefficacy of RNAi in B. mori and Manduca sexta, respectively (Garbutt and Reynolds, 2012; Swevers et al., 2011). In C. elegans, the relative inefficiency of neuronal RNAi appears mainly due to the low expression levels of the ce-sid1 dsRNA-transporter and high expression levels of the ce-eri1 dsRNAse (Calixto et al., 2010; Kennedy et al., 2004). In the present study, we have assessed the transcript levels of sid1 and eri1 in various tissues of the desert locust, but no tissue-dependent differences were observed (Fig. 4).

This article describes for the first time in detail the systemic RNAi-response in the desert locust. From this study, we can conclude that the desert locust possesses one of the most sensitive and persistent systemic RNAi-responses reported in an insect, and demonstrate for the first time tissue-dependency of RNAi in a member of the Orthoptera. Moreover, two main components of the RNAi-process are identified and a correlation between their transcript levels and the tissue-dependent RNAi-potency in the desert locust is described.

\section{Acknowledgements}

The authors gratefully thank J. Van Duppen, B. Deweer and F. Van Lommel for technical support; E. Marchal, L. Badisco and G. Perée for help with the dissections; R. Jonckers for taking care of the locust cultures and all lab members who took care of the locust cultures on holidays and in the weekends. In addition, the authors gratefully acknowledge the K.U. Leuven Research Foundation (GOA/ 11/02), the Research Foundation of Flanders (FWO-Flanders) and the Interuniversity Attraction Poles programme (Belgian Science Policy Grant P6/14) for financial support.

\section{Appendix A. Supplementary data}

Supplementary data related to this article can be found at http:// dx.doi.org/10.1016/j.ibmb.2012.09.004.

\section{References}

Badisco, L., Huybrechts, J., Simonet, G., Verlinden, H., Marchal, E., Huybrechts, R. Schoofs, L., De Loof, A., Vanden Broeck, J., 2011a. Transcriptome analysis of the desert locust central nervous system: production and annotation of a Schistocerca gregaria EST database. PLoS One 6 (3), e17274.

Badisco, L., Marchal, E., Van Wielendaele, P., Verlinden, H., Vleugels, R., Vanden Broeck, J., 2011b. RNA interference of insulin-related peptide and neuroparsins affects vitellogenesis in the desert locust Schistocerca gregaria. Peptides 32 (3), $573-580$.

Baum, J.A., Bogaert, T., Clinton, W., Heck, G.R., Feldmann, P., Ilagan, O., Johnson, S., Plaetinck, G., Munyikwa, T., Pleau, M., Vaughn, T., Roberts, J., 2007. Control of coleopteran insect pests through RNA interference. Nature Biotechnology 25 (11), 1322-1326.

Belles, X., 2010. Beyond Drosophila: RNAi in vivo and functional genomics in insects. Annual Review of Entomology 55, 111-128.

Calixto, A., Chelur, D., Topalidou, I., Chen, X.Y., Chalfie, M., 2010. Enhanced neuronal RNAi in C. elegans using SID-1. Nature Methods 7 (7), 554. U102.

Carthew, R.W., Sontheimer, E.J., 2009. Origins and mechanisms of miRNAs and siRNAs. Cell 136 (4), 642-655.

Czech, B., Zhou, R., Erlich, Y., Brennecke, J., Binari, R., Villalta, C., Gordon, A., Perrimon, N., Hannon, G.J., 2009. Hierarchical rules for Argonaute loading in Drosophila. Molecular Cell 36 (3), 445-456.

Dong, Y., Friedrich, M., 2005. Nymphal RNAi: systemic RNAi mediated gene knockdown in juvenile grasshopper. BMC Biotechnology 5, 25.

Garbutt, J.S., Reynolds, S.E., 2012. Induction of RNA interference genes by doublestranded RNA; implications for susceptibility to RNA interference. Insect Biochemistry and Molecular Biology 42 (9), 621-628.

Hammond, S.M., Boettcher, S., Caudy, A.A., Kobayashi, R., Hannon, G.J., 2001. Argonaute2, a link between genetic and biochemical analyses of RNAi. Science 293 (5532), 1146-1150.

Kennedy, S., Wang, D., Ruvkun, G., 2004. A conserved siRNA-degrading RNase negatively regulates RNA interference in C. elegans. Nature 427 (6975), 645649.

Kim, K., Lee, Y.S., Harris, D., Nakahara, K., Carthew, R.W., 2006. The RNAi pathway initiated by Dicer-2 in Drosophila. Cold Spring Harbor Symposia on Quantitative Biology 71, 39-44.

Lee, Y.S., Nakahara, K., Pham, J.W., Kim, K., He, Z., Sontheimer, E.J., Carthew, R.W. 2004. Distinct roles for Drosophila Dicer-1 and Dicer-2 in the siRNA/miRNA silencing pathways. Cell 117 (1), 69-81.

Luo, Y., Wang, X., Yu, D., Kang, L., 2012. The SID-1 double-stranded RNA transporter is not required for systemic RNAi in the migratory locust. RNA Biology 9 (5).

Mao, Y.B., Cai, W.J., Wang, J.W., Hong, G.J., Tao, X.Y., Wang, L.J., Huang, Y.P., Chen, X.Y. 2007. Silencing a cotton bollworm P450 monooxygenase gene by plantmediated RNAi impairs larval tolerance of gossypol. Nature Biotechnology 25 (11), 1307-1313.

Marchal, E., Verlinden, H., Badisco, L., Van Wielendaele, P., Vanden Broeck, J., 2012. RNAi-mediated knockdown of Shade negatively affects ecdysone-20hydroxylation in the desert locust, Schistocerca gregaria. Journal of Insect Physiology 58 (7), 890-896.

Marchal, E., Badisco, L., Verlinden, H., Vandersmissen, T., Van Soest, S., Van Wielendaele, P., Vanden Broeck, J., 2011a. Role of the Halloween genes, Spook and Phantom in ecdysteroidogenesis in the desert locust, Schistocerca gregaria. Journal of Insect Physiology 57 (9), 1240-1248.

Marchal, E., Zhang, J., Badisco, L., Verlinden, H., Hult, E.F., Van Wielendaele, P., Yagi, K.J., Tobe, S.S., Vanden Broeck, J., 2011b. Final steps in juvenile hormone biosynthesis in the desert locust, Schistocerca gregaria. Insect Biochemistry and Molecular Biology 41 (4), 219-227.

Miller, S.C., Brown, S.J., Tomoyasu, Y., 2008. Larval RNAi in Drosophila? Development Genes and Evolution 218 (9), 505-510.

Ott, S.R., Verlinden, H., Rogers, S.M., Brighton, C.H., Quah, P.S., Vleugels, R.K. Verdonck, R., Vanden Broeck, J., 2012. Critical role for protein kinase A in the acquisition of gregarious behavior in the desert locust. Proceedings of the National Academy of Sciences of the United States of America 109(7),E381-E387.

Pattyn, F., Speleman, F., De Paepe, A., Vandesompele, J., 2003. RTPrimerDB: the realtime PCR primer and probe database. Nucleic Acids Research 31 (1), 122-123.

Saleh, M.C., van Rij, R.P., Hekele, A., Gillis, A., Foley, E., O'Farrell, P.H., Andino, R. 2006. The endocytic pathway mediates cell entry of dsRNA to induce RNAi silencing. Nature Cell Biology 8 (8), 793-802.

Schiebel, W., Pelissier, T., Riedel, L., Thalmeir, S., Schiebel, R., Kempe, D., Lottspeich, F. Sanger, H.L., Wassenegger, M., 1998. Isolation of an RNA-directed RNA polymerase-specific cDNA clone from tomato. The Plant Cell 10 (12), 2087-2101.

Sijen, T., Fleenor, J., Simmer, F., Thijssen, K.L., Parrish, S., Timmons, L., Plasterk, R.H., Fire, A., 2001. On the role of RNA amplification in dsRNA-triggered gene silencing. Cell 107 (4), 465-476.

Swevers, L., Liu, J., Huvenne, H., Smagghe, G., 2011. Search for limiting factors in the RNAi pathway in silkmoth tissues and the Bm5 cell line: the RNA-binding proteins R2D2 and Translin. PLoS One 6 (5), e20250.

Terenius, O., Papanicolaou, A., Garbutt, J.S., Eleftherianos, I., Huvenne, H., Kanginakudru, S., Albrechtsen, M., An, C., Aymeric, J.L., Barthel, A., Bebas, P., Bitra, K., Bravo, A., Chevalier, F., Collinge, D.P., Crava, C.M., de Maagd, R.A Duvic, B., Erlandson, M., Faye, I., Felfoldi, G., Fujiwara, H., Futahashi, R., Gandhe, A.S., Gatehouse, H.S., Gatehouse, L.N., Giebultowicz, J.M., Gomez, I., Grimmelikhuijzen, C.J., Groot, A.T., Hauser, F., Heckel, D.G., Hegedus, D.D., 
Hrycaj, S., Huang, L., Hull, J.J., Iatrou, K., Iga, M., Kanost, M.R., Kotwica, J., Li, C., Li, J., Liu, J., Lundmark, M., Matsumoto, S., Meyering-Vos, M., Millichap, P.J., Monteiro, A., Mrinal, N., Niimi, T., Nowara, D., Ohnishi, A., Oostra, V., Ozaki, K. Papakonstantinou, M., Popadic, A., Rajam, M.V., Saenko, S., Simpson, R.M. Soberon, M., Strand, M.R., Tomita, S., Toprak, U., Wang, P., Wee, C.W., Whyard, S Zhang, W., Nagaraju, J., Ffrench-Constant, R.H., Herrero, S., Gordon, K Swevers, L., Smagghe, G., 2011. RNA interference in Lepidoptera: an overview of successful and unsuccessful studies and implications for experimental design. Journal of Insect Physiology 57 (2), 231-245.

Tomari, Y., Du, T., Zamore, P.D., 2007. Sorting of Drosophila small silencing RNAs. Cell $130(2), 299-308$

Tomari, Y., Zamore, P.D., 2005. Perspective: machines for RNAi. Genes \& Development 19 (5), 517-529.

Tomoyasu, Y., Miller, S.C., Tomita, S., Schoppmeier, M., Grossmann, D., Bucher, G. 2008. Exploring systemic RNA interference in insects: a genome-wide survey for RNAi genes in Tribolium. Genome Biology 9 (1), R10.

Ulvila, J., Parikka, M., Kleino, A., Sormunen, R., Ezekowitz, R.A., Kocks, C., Ramet, M., 2006. Double-stranded RNA is internalized by scavenger receptor-mediated endocytosis in Drosophila S2 cells. The Journal of Biological Chemistry 281 (20), 14370-14375.

Van Hiel, M.B., Van Wielendaele, P., Temmerman, L., Van Soest, S., Vuerinckx, K., Huybrechts, R., Vanden Broeck, J., Simonet, G., 2009. Identification and validation of housekeeping genes in brains of the desert locust Schistocerca gregaria under different developmental conditions. BMC Molecular Biology 10, 56

Van Wielendaele, P., Dillen, S., Marchal, E., Badisco, L., Vanden Broeck, J., 2012. CRFlike diuretic hormone negatively affects both feeding and reproduction in the desert locust, Schistocerca gregaria. PLoS One 7 (2), e31425.

Whyard, S., Singh, A.D., Wong, S., 2009. Ingested double-stranded RNAs can act as species-specific insecticides. Insect Biochemistry and Molecular Biology 39 (11), $824-832$.

Winston, W.M., Molodowitch, C., Hunter, C.P., 2002. Systemic RNAi in C. elegans requires the putative transmembrane protein SID-1. Science 295 (5564), 24562459.

Zhou, R., Hotta, I., Denli, A.M., Hong, P., Perrimon, N., Hannon, G.J., 2008. Comparative analysis of argonaute-dependent small RNA pathways in Drosophila. Molecular Cell 32 (4), 592-599. 